\section{Unilateral recession- resection in children with exotropia of the convergence insufficiency type}

\begin{abstract}
Purpose The surgical success rates for intermittent exotropia of the convergence insufficiency type have been reported to be variable, and most were studied retrospectively in adults. The purpose of this study was to evaluate prospectively the long-term surgical results of unilateral lateral rectus (LR) muscle recession and medial rectus (MR) muscle resection in children with intermittent exotropia of the convergence insufficiency type.

Methods A total of 14 children with intermittent exotropia greater at near than at distance by 10 prism diopters (PD) or more were included in this prospective study. The amounts of resection and recession were based on near and distance deviation, respectively. Minimum follow-up was 1 year (mean 26.6 months; range, 12-68 months) after surgery. The paired $t$-test was used to compare preoperative and postoperative measurements of the angle of deviation at distance and near, near-distance difference.
\end{abstract}

Results Significant postoperative reduction was achieved in terms of mean distance exodeviation, from 22.5 PD to 9.1 PD $(P=0.000)$, and mean near exodeviation from 33.8 PD to 13.6 PD $(P=0.000)$. Mean neardistance difference reduced from 11.3 PD preoperatively to $4.6 \mathrm{PD}$ postoperatively $(P=0.000)$. Fresnel prism was used temporarily to treat postoperative esotropia in only one patient for postoperative 6 months. Conclusions Unilateral surgery biased to MR strengthening more than LR weakening in children with intermittent exotropia of the convergence insufficiency type, was found to successfully reduce both distance and near deviation and to collapse near-distance differences with a low risk of long-term postoperative esotropia.

Eye (2007) 21, 344-347. doi:10.1038/sj.eye.6702197; published online 2 December 2005

Keywords: exotropia; convergence insufficiency; recession and resection

\section{Introduction}

The convergence insufficiency type of exotropia is characterized by a greater exodeviation at near than at distance, usually by 10 prism diopters (PD) or more. ${ }^{1-4}$ The surgical procedures recommended for this form of exotropia include bilateral lateral rectus (LR) muscle recession with or without a slanting procedure, bilateral medial rectus (MR) muscle resection with or without a slanting procedure, $\mathrm{LR}$ recession with $\mathrm{MR}$ resection, or MR resection with an adjustable suture. ${ }^{1,2,5-11}$ Surgical success rates have been reported to vary between 18 and $92 \% .^{1,2,5-11}$

The majority of previous studies have been performed in adults with intermittent exotropia $(X(T))$ of the convergence insufficiency type. ${ }^{1,6-11}$ The convergence insufficiency type is much less frequent in children with $\mathrm{X}(\mathrm{T})$ and surgical results have rarely been reported. Kraft et $a l^{5}$ described a technique wherein the monocular strengthening of MR resection exceeds LR weakening; the amount of MR resection used was based on near deviation, and the amount of LR recession was based on far deviation. In all patients, near deviation was corrected and near-distance difference was significantly reduced. However, the mean age of the patients treated was 28 years and the study included only three children among 14 patients. Thus, we undertook this study to evaluate the long-term 
results of unilateral surgery as described by Kraft et $a l^{5}$ as a primary surgery, without an adjustable suture, for children with $\mathrm{X}(\mathrm{T})$ of the convergence insufficiency type.

\section{Methods}

In this prospective study, we included 14 consecutive children (six boys and eight girls) with $X(T)$ of the convergence insufficiency type. All cases had X(T) greater at near than at distance by 10 PD or more, which was confirmed at repeated examinations at intervals of at least 1 month. No patient with previous ocular or extraocular muscle surgery, ocular or neurologic pathologic conditions, amblyopia, or a postoperative follow-up of $<1$ year was included in the study. Informed consent was obtained from the parents of all participants before operation according to a protocol approved by the institutional review board for the protection of human subjects (declaration of Helsinki).

The symptoms of convergence insufficiency type $\mathrm{X}(\mathrm{T})$ were outward deviation in all 14 patients, photophobia in four patients (case Nos. 1, 4-6), and diplopia in one patient (case No. 2). None of the patients had worn prism glasses. Push-up training was attempted to build convergence fusional amplitude, but no change in angle of deviation or symptoms occurred.

The children ranged in age from 5 to 10 years (mean, 7.1 years), and the mean length of follow-up was 26.6 months (range, 12-68 months). Table 1 itemizes patient data, that is, age, sex, preoperative deviations, binocularity, surgeries performed, postoperative alignments, and binocularity at the last follow-up for all children. All patients underwent full ophthalmologic and orthoptic evaluations. Deviations were measured with prism and alternating cover tests while patients fixated on accommodation-controlling targets at 6 and $1 / 3 \mathrm{~m}$. An additional near measurement was obtained after $1 \mathrm{~h}$ of monocular occlusion of the habitually deviating eye. Myopia $>1.00$ diopter (D), astigmatism $>1.50 \mathrm{D}$ and hyperopia $>3.00 \mathrm{D}$ were usually treated with glasses which fully corrected the myopia or astigmatism, and $1.00-1.50 \mathrm{D}$ less than full cycloplegic hyperopic refraction before a final surgical decision was made.

Abnormalities in duction and version were checked and graded on a scale of 0 (normal) to -4 (inability to move into the field of action). Stereopsis was evaluated using the Titmus test at near and at fusion using the Worth four-dot test. We explained to parents before operation that alternate patching or prism therapy would be required in cases of postoperative overcorrection at distance and/or at near.

Unilateral surgery was performed on the more commonly deviating eye. The amounts of recession and resection were based on the distance and near deviation, respectively, according to preoperative deviation of $X(T)$.

Postoperative follow-up intervals were determined according to patient status, but examinations were usually scheduled at 1 day, 1 week, and at 1, 3, 6, and 12 months after surgery, and annually thereafter. Postoperatively, alternate patching was performed in cases that manifested consecutive esotropia with diplopia at distance and/or near. Fresnel prisms were used in consecutive esotropia refractory to alternating patching. Alternate patching or prisms were discontinued as soon as the patients maintained single binocular vision.

Table 1 Database for 14 children treated

\begin{tabular}{|c|c|c|c|c|c|c|c|c|c|}
\hline \multirow[t]{2}{*}{ Case No. } & \multirow{2}{*}{$\begin{array}{c}\text { Age } \\
\text { (years)/Sex }\end{array}$} & \multicolumn{3}{|c|}{ Preoperative results } & \multirow{2}{*}{$\begin{array}{c}\text { Surgery LR } \\
\text { Rec/MR Res }(\mathrm{mm})\end{array}$} & \multicolumn{3}{|c|}{ Postoperative results } & \multirow{2}{*}{$\begin{array}{l}\text { Follow-up } \\
\text { (month) }\end{array}$} \\
\hline & & $\begin{array}{c}\text { XT pattern } \\
D / N(P D)\end{array}$ & $\begin{array}{l}\text { Difference, } \\
N-D(P D)\end{array}$ & $\begin{array}{l}\text { Stereopsis }^{a} / \\
\text { Fusion }\end{array}$ & & $\begin{array}{c}\text { Pattern } \\
D / N(P D)\end{array}$ & $\begin{array}{l}\text { Difference, } \\
N-D(P D)\end{array}$ & $\begin{array}{l}\text { Stereopsis/ } \\
\text { fusion }\end{array}$ & \\
\hline 1 & $8 / \mathrm{M}$ & $20 / 35$ & 15 & $80 /-$ & $5 / 6$ & ortho/8 XT & 8 & $80 /+$ & 23 \\
\hline 2 & $6 / \mathrm{M}$ & $20 / 35$ & 15 & $50 /+$ & $5 / 6$ & $6 \mathrm{XT} / 10 \mathrm{XT}$ & 4 & $40 /+$ & 38 \\
\hline 3 & $5 / \mathrm{M}$ & $20 / 30$ & 10 & $400 /-$ & $5 / 5.5$ & $8 \mathrm{XT} / 8 \mathrm{XT}$ & 0 & $200 /-$ & 68 \\
\hline 4 & $7 / \mathrm{F}$ & $20 / 35$ & 15 & $100 /+$ & $5 / 6$ & $8 \mathrm{XT} / 6 \mathrm{XT}$ & -2 & $50 /+$ & 34 \\
\hline 5 & $8 / \mathrm{M}$ & $25 / 35$ & 10 & $100 /+$ & $6 / 5$ & $12 \mathrm{XT} / 20 \mathrm{XT}$ & 8 & $200 /-$ & 45 \\
\hline 6 & $7 / \mathrm{M}$ & $30 / 43$ & 13 & $80 /-$ & $6.5 / 6.5$ & $20 \mathrm{XT} / 28 \mathrm{XT}$ & 8 & $60 /+$ & 12 \\
\hline 7 & $7 / \mathrm{F}$ & $30 / 40$ & 10 & $100 /-$ & $6.5 / 6.5$ & $15 \mathrm{XT} / 25 \mathrm{XT}$ & 10 & $80 /+$ & 12 \\
\hline 8 & $7 / F$ & $20 / 30$ & 10 & $80 /-$ & $5 / 5.5$ & Ortho/ortho & 0 & $40 /+$ & 12 \\
\hline 9 & $6 / F$ & $15 / 25$ & 10 & $50 /+$ & $4 / 5$ & $15 \mathrm{XT} / 20 \mathrm{XT}$ & 5 & $40 /+$ & 12 \\
\hline 10 & $9 / \mathrm{M}$ & $15 / 25$ & 10 & $40 /+$ & $4 / 5$ & $10 \mathrm{XT} / 15 \mathrm{XT}$ & 5 & $40 /+$ & 60 \\
\hline 11 & $6 / F$ & $25 / 35$ & 10 & $800 /-$ & $6 / 6$ & $6 \mathrm{ET} / 6 \mathrm{ET}$ & 0 & $100 /+$ & 12 \\
\hline 12 & $7 / \mathrm{F}$ & $30 / 40$ & 10 & $50 /-$ & $6.5 / 6.5$ & $12 \mathrm{XT} / 20 \mathrm{XT}$ & 8 & $50 /+$ & 12 \\
\hline 13 & $10 / \mathrm{F}$ & $20 / 35$ & 15 & $40 /+$ & $5 / 6$ & 15XT/20XT & 5 & $140 /-$ & 12 \\
\hline 14 & $10 / \mathrm{F}$ & $25 / 35$ & 10 & $40 /+$ & $6 / 6$ & 12XT/18XT & 6 & $60 /+$ & 20 \\
\hline
\end{tabular}

a Seconds of arc; $\mathrm{LR} \mathrm{Rec}=$ lateral rectus recession; MR Res $=$ medial rectus resection; $\mathrm{D} / \mathrm{N}=$ distance $/$ near; $\mathrm{N}-\mathrm{D}=$ near minus distance; $\mathrm{PD}=$ prism diopters; $\mathrm{XT}=$ exodeviation; $-=$ no existence of fusion; ortho = orthophoria, $\mathrm{ET}=$ esodeviation. 
A successful outcome was defined as a postoperative residual deviation at near and distance of $\leq 10 \mathrm{PD}$ and a near-distance exodeviation difference of $\leq 10 \mathrm{PD}$.

The paired $t$-test was used to compare; mean distance angle of deviation preoperatively and postoperatively, mean near angle of deviation preoperatively and postoperatively, and mean near-distance exodeviation difference preoperatively and postoperatively. In addition, mean postoperative deviated angles at each scheduled follow-up were calculated and also compared using the paired $t$-test. The Microsoft Excel (version 5.00, 2005) program was used for all statistical calculations.

\section{Results}

The postoperative alignments and binocularity at last follow-up visits and the duration of follow-up for the 14 children are listed in Table 1. Before surgery, mean exodeviation was 22.5 PD (range, 25-43 PD) at distance, and 33.8 PD (range, 15-30 PD) at near. At the last followup, mean exodeviation at distance was 9.1 PD (range, -6 to $20 \mathrm{PD}$ ) and at near, 13.6 PD (range, -6 to $28 \mathrm{PD}$ ). Significant postoperative reductions were observed in terms of exodeviations at distance $(P<0.001)$, and at near $(P<0.001)$. Near-distance differences reduced from a preoperative mean of $11.3 \mathrm{PD}$ (range, 10 to $15 \mathrm{PD}$ ) to a postoperative mean of $4.6 \mathrm{PD}$ (range, -2 to $10 \mathrm{PD}$ ), which was a significant reduction $(P<0.001)$.

At the final follow-up examination, overall success was achieved in $42.9 \%$. The eight patients that did not meet the surgical success criteria had recurrent exotropia. However, near-distance differences in these cases with recurrent exotropia collapsed to $<10 \mathrm{PD}$.

On postoperative day 1 , no patient showed abduction limitation in the operated eye. However, 10 of the 14 children $(71 \%)$ had esodeviations at distance and/or at near ranging from 4 to $30 \mathrm{PD}$. Most of them resolved; at 1 week in three, 2 weeks in two, and at 1 month in four. One patient (No. 4) had 8 PD of esotropia at distance and $12 \mathrm{PD}$ at near with diplopia. Therefore, this patient was used base-out fresnel prisms and was able to discontinue prism therapy until 6 months postoperatively.

The greatest amounts of exodrift observed during the first postoperative month are shown in Figure 1.

Thereafter, no statistical significance was found between mean deviated angles at distance or at near.

Stereopsis at near improved postoperatively rather than preoperatively in eight patients. Six of seven patients who preoperatively lost fusional ability achieved function at distance or at near fusion postoperatively.

\section{Discussion}

Reported symptoms of convergence insufficiency type $X(T)$ include headache, diplopia, blur, and asthenopia in

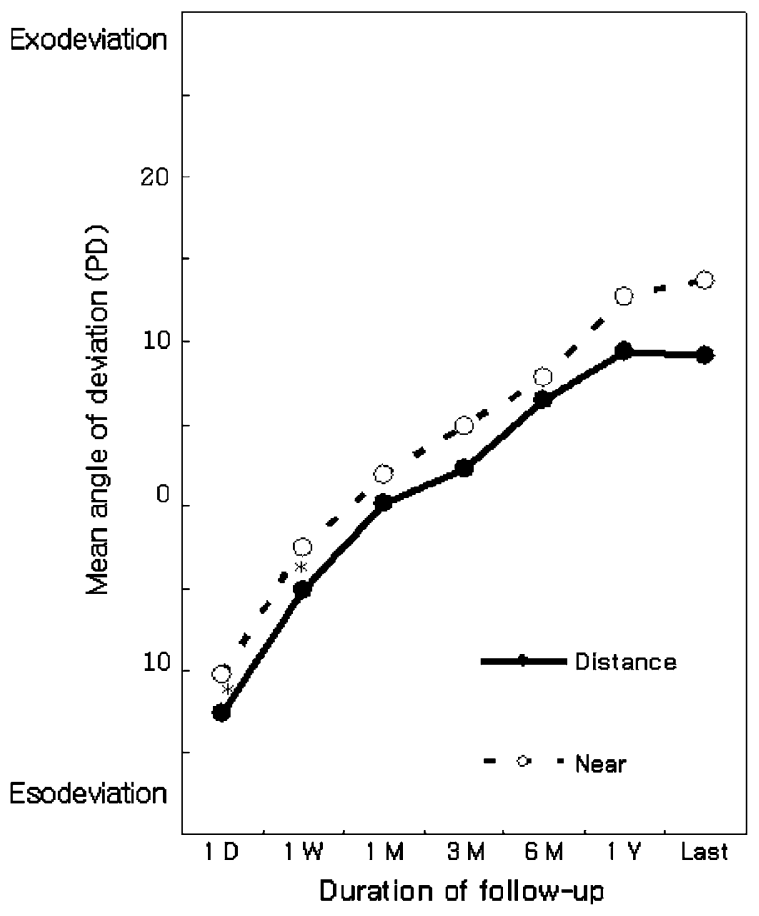

Figure 1 Postoperative stability of deviated angle in treated children. Greatest amounts of exo-drift at distance and at near were observed during the first postoperative month $\left({ }^{*} P\right.$ value of distance angles between postoperative day 1 and postoperative week 1 was $<0.05$ ).

adults. ${ }^{3,4,7-9,12}$ In contrast, symptoms documented in the present study deviated from those reported previously. The probable cause for this discrepancy is that different criteria were used for patient recruitment. We only included children who had exotropia with convergence insufficiency, whereas other studies included patients with a history of prolonged difficulties with near work and had undergone extended periods of unsuccessful nonsurgical therapy. ${ }^{5,7-9}$ Korean children of mean age 7.1 years were included in the present study, whereas the mean ages of patients in other reports ranges from 15 to 51 years. ${ }^{1,6-8}$ This age discrepancy is important in selecting surgical methods, because children are susceptible to amblyopia due to postoperative overcorrection, and they are not cooperative enough to undergo adjustable suture surgery. To date, no study has been conducted on the surgical outcomes of $\mathrm{XT}$ children with convergence insufficiency.

The several procedures have been recommended to date for convergence insufficiency type of exotropia but reported surgical results are variable. ${ }^{1,25-11} \mathrm{Kraft}$ et al ${ }^{5}$ described a technique wherein the monocular strengthening of MR resection exceeds the weakening of LR. In all patients, near deviation was corrected and near-distance difference was significantly reduced. Compared to the results presented in their report, the 
surgical results of present study are unsatisfactory. However, in their study five of their 14 patients were reoperation cases and adjustable sutures were performed in five cases (primary surgery in three cases, and reoperation in two cases). Thus, we believe that the different inclusion criteria used may have caused the difference of surgical result.

It has been reported that $X(T)$ shows a strong tendency to recur and drift into permanent exotropia with time after surgery. ${ }^{13-18}$ Postoperative success rates vary from 41 to $95 \%$ in previous reports, which may be explained by different follow-up lengths., ${ }^{2,13-15}$ A general drift in postoperative alignment with time was also demonstrated by our study (Figure 1). Therefore, success rates cannot be compared between the study of Kraft et $a l^{5}$ and the present study because of differences in the follow-up period (mean, 7.9 months ${ }^{5}$ vs 26.6 months in the present study).

Several authors have reported postoperative diplopia in convergence insufficiency exotropia. ${ }^{6-8}$ Hermann $^{7}$ reported 14 patients treated by bilateral MR resection. Postoperatively fresnel prisms were used in all of these patients, and time to esotropia and diplopia resolution at distance averaged 2.7 months. von Noorden ${ }^{6}$ reported on six patients, who received bilateral MR resections; fresnel prisms were required to treat diplopia at distance in five patients for 5 weeks, and one patient for 5 months. Choi and Rosenbaum ${ }^{8}$ performed unilateral or bilateral MR resection with an adjustable suture with a target angle of 10-20 PD esotropia at distance and 5-10 PD esotropia at near. Postoperatively fresnel prisms were used in 14 of 21 cases. In all, 11 cases were able to discontinue prism use at 3 months after surgery, whereas the other three cases required prisms for 6 months or over. Compared to the frequency of postoperative diplopia in above reports, Kraft $e t a l^{5}$ reported that unilateral surgery biased toward MR strengthening and geared to near deviation has a lower risk of creating a large postoperative esodeviation at distance. Similarly, we noted that postoperative esotropia and diplopia were short-lived ( $<4$ weeks), except in one patient. Therefore, we find that this approach is particularly useful in children with a low risk of esodeviation.

In conclusion, the present study indicates that for exotropic children with near-distance differences of $\geq 10$ PD, unilateral surgery biased toward MR strengthening improves postoperative results and reduces near-distance differences without the risk of long-term postoperative diplopia.

\section{References}

1 Snir M, Axer-Siegel R, Shalev B, Sherf I, Yassur Y. Slanted lateral rectus recession for exotropia with convergence weakness. Ophthalmology 1999; 106: 992-996.

2 Raab EL, Parks MM. Recession of the lateral recti; effect of preoperative fusion and distance-near relationship. Arch Ophthalmol 1975; 93: 584-586.

3 Kushner BJ, Morton GV. Distance/near differences in intermittent exotropia. Arch Ophthalmol 1998; 116: 478-486.

4 Burian HM. Exodeviations; their classification, diagnosis and treatment. Am J Ophthalmol 1966; 62: 1161-1166.

5 Kraft SP, Levin AV, Enzenauer RW. Unilateral surgery for exotropia with convergence weakness. J Pediatr Ophthalmol Strabismus 1995; 32: 183-187.

6 von Noorden GK. Resection of both medial rectus muscles in organic convergence insufficiency. Am J Ophthalmol 1976; 81: 223-226.

7 Hermann JS. Surgical therapy for convergence insufficiency. J Pediatr Ophthalmol Strabismus 1981; 18: 28-31.

8 Choi DG, Rosenbaum AL. Medial rectus resection(s) with adjustable suture for intermittent exotropia of the convergence insufficiency type. J AAPOS 2001; 5: 13-17.

9 Nemet P, Stolovich C. Biased resection of the medial recti: a new surgical approach to convergence insufficiency. Binocul Vis Strabismus Q 1990; 5: 213-216.

10 Biedner B. Treatment of convergence insufficiency by single medial rectus muscle slanting resection. Ophthalmic Surg Lasers 1997; 28: 347-348.

11 Haldi BA. Surgical management of convergence insufficiency. Am Orthopt J 1978; 28: 106-109.

12 Daum KM. Convergence insufficiency. Am J Optom Physiol Opt 1984; 61: 16-22.

13 Beneish R, Flanders M. The role of stereopsis and early postoperative alignment in long-term surgical results of intermittent exotropia. Can J Ophthalmol 1994; 29: 119-124.

14 Scott WE, Keech R, Mash AJ. The postoperative results and stability of exodeviations. Arch Ophthalmol 1981; 99: 1814-1818.

15 Stoller SH, Simon JW, Lininger LL. Bilateral lateral rectus recession for exotropia: a survival analysis. J Pediatr Ophthalmol Strabismus 1994; 31: 89-92.

16 Maruo T, Kubota N, Sakaue T, Usui C. Intermittent exotropia surgery in children: long term outcome regarding changes in binocular alignment. A study of 666 cases. Binocul Vis Strabismus Q 2001; 16: 265-270.

17 Pratt-Johnson J, Wee HS. Suppression associated with exotropia. Can J Ophthalmol 1969; 4: 136-144.

18 Richard JM, Parks MM. Intermittent exotropia. Surgical results in different age groups. Ophthalmology 1983; 90: 1172-1177. 